\title{
A condition monitoring system for an early warning of developing faults in wind turbine electrical systems
}

\author{
Xiandong Ma, P Cross and Peng Qian
}

\begin{abstract}
Electrical condition monitoring (CM) normally involves the collection of high-frequency, instantaneous data for feature extraction. This paper presents a novel development of an electrical condition monitoring system for wind turbines. The system is developed based upon a control and data acquisition system, for which hardware modules can be configured for a particular set of signals, thus tailoring the system to a specific range of monitoring tasks. A wavelet-based singularity detection method is proposed, which automatically calculates the Lipschitz exponent, a measure to describe the local transient activities in the measurement signal. The relationship between the Lipschitz exponent and the type and severity of faults occurring on the grid and in the power electronics is explored. The proposed algorithms are tested and validated using simulation data from computer simulations of a doubly-fed induction generator (DFIG) wind turbine with a grid connection. A field-programmable gate array (FPGA) embedded in the system has been utilised, allowing the signal processing tasks to be undertaken in real-time for monitoring purposes. The paper demonstrates that a fault signal of small magnitude generated at the early stage of a fault carries the same Lipschitz exponent as the signal of large magnitude generated at the late stage of the fault, thereby providing an early warning before the fault develops into a
\end{abstract} detrimental one.

Keywords: reliability, condition monitoring, operation and maintenance, real-time simulation, wind turbines.

\section{Introduction}

The published statistics state that, for wind turbines in most European countries, the failure rate per turbine per year is as high as 3.5 for larger units (>1 MW) and around 1.0 for smaller units $(<500 \mathrm{~kW})^{[1]}$. Over an operating life of 20 years, maintenance costs for an onshore wind farm are estimated to be $10-15 \%$ of the total income and for an offshore wind turbine farm over the same period these costs are estimated to be $20-25 \%$ of the total income $e^{[2,3]}$. In this regard, the monitoring and diagnostics of turbines play an increasingly important role in the competitive operation of wind farms. Consolidated knowledge about the past and current condition of wind turbines can be used to improve performance, reliability and availability, thus enabling the optimal scheduling of maintenance activities and minimising the risk of costly, unexpected failures of turbines during their service life.

Typically, CM systems comprise sensors, data acquisition, data filtration, data storage, feature extraction, pattern recognition and decision-making units. A review of condition monitoring of rotating electrical machines can be found $i^{[4]}$ and a review of the design and system architecture of condition monitoring systems for wind turbines can be found $\mathrm{in}^{[5]}$. The majority of vibration monitoring systems available measure vibration, which requires a range of sensors for different frequencies ${ }^{[6]}$. Other systems measure parameters such as blade stress and the temperatures of the nacelle, coolant, oil and generator ${ }^{[7]}$. Monitoring data may be stored locally or transferred to a central computer for further diagnosis. The large number of data generated requires techniques such as fast Fourier transform (FFT) and enveloped FFT to reveal patterns in the data, which might infer the nature, form and extent of the degradation of components ${ }^{[8]}$.

Electrical condition monitoring normally involves the analysis

\section{Submitted 25.06.16 / Accepted 27.10.16}

Xiandong $\mathrm{Ma}$ is a senior lecturer in the Engineering Department at Lancaster University, UK. He received a BEng degree in electrical engineering in 1986, an MSc degree in power systems and automation in 1989 and a PhD in partial discharge-based high-voltage condition monitoring in 2003. His research interests include intelligent condition monitoring and fault diagnosis of distributed generation systems in wind turbines, with an emphasis on analytical and experimental investigation, advanced signal processing, data mining and instrumentation and electromagnetic NDT testing and imaging.

Philip Cross received his BSc degree in geophysical sciences from Lancaster University, UK, in 1991 and his MSc degree in information technology from the University of Teesside, UK, in 1992. Following severalyears in industry, Dr Cross returned to Lancaster University, where he obtained an MSc degree in informatics and a PhD in engineering in 2008 and 2012, respectively. He worked as a research associate in the Engineering Department at Lancaster University from 2012 to 2013. His research interests include data-based modelling, feedback control, system identification, artificial intelligence, condition monitoring and renewable energy.

Peng Qian* received a BEng degree in electrical engineering and automation in 2009 and an MSc degree in power electronics and drives in 2014, both from Jiangsu University, China. He has been pursuing his PhD studies in condition monitoring of wind turbines at Lancaster University, UK, since January 2015. His research interests include predictive condition monitoring, data driven-based modelling, optimal energy management and hardware-in-the-loop testing.

The authors are with the Engineering Department, Lancaster University, Lancaster LA1 4YW, UK. Email: xiandong.ma@lancaster. ac.uk/philipcross68@outlook.com / p.qian@lancaster.ac.uk

*Corresponding author. Tel: +44 (0)1524 593700; Fax: +44 (0) 1524381707 
of transient electrical behaviours of the components or systems and high-frequency sampling is therefore required. For example, in insulation condition monitoring based on partial discharge (PD) measurement and analysis, a sampling rate usually in the range of $\mathrm{MHz}$ is required in order to capture the magnitude of the discharges and the number of discharges with regard to a particular phase angle within an AC cycle for an accurate PD pattern recognition ${ }^{[9]}$.

Wind turbines usually operate at varying speeds and are subject to intermittent and stochastic aerodynamic effects. In addition, power electronics are often required to control the flow of power between the wind turbine and the local grid system in either direction, depending on the load and climatic conditions; hence, the behaviour of the measurement signals is non-stationary. Consequently, recent research has focused on the use of windowed Fourier transform and wavelet analysis to cope with the non-stationary components in the measurement data ${ }^{[9,10]}$. Research into CM techniques has also employed generic software and hardware for monitoring and analysis ${ }^{[11,12]}$. However, it should be noted that, at present, many $\mathrm{CM}$ systems use individual monitoring modules for a specific component or subsystem with a particular type of detection technique. Clearly, it would be beneficial to monitor parameters associated with a number of components to better identify potential failures of individual components and of the system as a whole ${ }^{[13]}$.

This paper proposes a novel system for electrical condition monitoring of wind turbines, although the system is designed such that it can use a variety of data acquisition modules to perform CM tasks for specific components. An early warning algorithm based on wavelet singularity analysis is developed to identify the faults occurring on the grid and in the power electronics when they are still at an incipient stage. A grid-connected DFIG wind turbine has been simulated to generate useful data at a system level to validate the proposed algorithms. In order to realise the proposed system, a control unit incorporating an FPGA module has been employed, allowing signal processing tasks to be carried out in real time.

\section{Overview of the system}

A key factor to take into account when considering condition monitoring is how measurements are realised and performed. Failures in wind turbines can occur in any subsystem of the turbine, from the blades, through the drivetrain to the generator. In addition, monitoring techniques need to deal with meteorological, acoustic, optical, mechanical, thermal, electromagnetic and electrical phenomena while generating data for collection and interpretation. Anomalies occurring in the turbine and the grid will adversely affect the output of the generator, such as current, voltage and power output, and hence the performance of the device.

\subsection{Configurable data acquisition system}

The proposed system is illustrated in Figure 1. A set of analogue input modules, controlled by a real-time control unit, are used to acquire signals from sensors that are physically connected to different subsystems of an operational wind turbine. Interactions between these different monitoring modules are taken into account; for example, the turbine speed could affect both the bearing temperature and shaft vibration levels of the generator. Monitoring data alone or, when necessary, together with relevant operational data collected by other in situ systems, such as a supervisory control and data acquisition (SCADA) system, are sent to the host computer via a local area network and saved to a historical database. These data can be transmitted to a remote support centre for further analysis.

The input modules can be configured for different sensors and various inputs. For example, an analogue module can be used for speed measurements, including wind, rotor and generator speed, while another is used for measurements of electrical parameters, such as current, voltage and power outputs. Further analogue modules can be used for vibration measurements. A thermocouple module can be added to monitor the temperature of components, such as the bearings, generator winding or nacelle, and control signals can be monitored with a digital input module. The CM system is controlled by a real-time control unit, which reads data from direct memory access first-in first-out (DMA-FIFO) buffers and also exchanges data over the internet through a transmission control protocol/internet protocol (TCP/IP) connection with a host PC, ie the CM server. Software modules are implemented on the host PC using LabVIEW for user interface options and data analysis. This modular structure can provide significant flexibility for monitoring parameters by adding new hardware modules and data analysis software as required.

In this system, an FPGA is employed primarily because of its reconfigurable I/O capability. The processing unit in the FPGA can be connected directly to the different types of acquisition hardware, therefore minimising latency. An FPGA consists of many small building blocks, including logic blocks, memories and I/O blocks, plus interconnecting wires and switches, the functions of which can be configured by the user. The blocks can work in parallel and can also be interconnected to build more complex operation blocks. These distinct features have made FPGA-based hardware systems very attractive for real-time signal processing applications ${ }^{[14-16]}$. The FPGA in this system employs three DMA channels to which data can be written concurrently, thereby allowing signal processing tasks to be carried out in real time.

Computer simulations of a DFIG wind turbine with a grid connection were created. Useful data at a system level under 
different grid and power electronic faults were obtained to validate the algorithms proposed in this paper. The model will be described in more detail in Section 3.

\subsection{Wavelet-based singularity detection}

It is well known that at the time instant when a fault occurs, the output signals usually contain transient changes or discontinuities. The analysis of non-stationary signals for appropriate feature extraction is normally performed by the Fourier transform and wavelet transform in the context of signal processing. Fourier analysis has been used extensively to analyse vibration signals ${ }^{[6]}$, in which the harmonics of signals generated by a healthy machine are compared to those of the signals generated by monitored machines. The frequency-related characteristic of these signals can be vital for abnormality diagnosis and discrimination. Furthermore, wavelet transform techniques have been researched to mine the nonstationary components for both time- and frequency-related local characteristics in the measurement data. For example, continuous wavelet transforms have been proposed for the detection of mechanical and electrical faults in drivetrains by tracking signal energy within particular frequency bands ${ }^{[10]}$, and the detection of rotor eccentricity by extracting the strength of particular frequency components ${ }^{[17]}$.

Intuitively, the changes in measured signals in the early stages of a fault are usually small in magnitude but may indicate the start of a significant failure. However, small magnitude signals are normally undetected by conventional methods, for example threshold rules, particularly in a harsh offshore environment. Despite being low in magnitude and of short duration, such a small signal may essentially carry the same features as a large signal.

The local singularities of a signal can be described by the Lipschitz exponent. It should be noted that the Fourier transform can only measure the global Lipschitz exponent of a signal, which cannot provide information regarding the time distribution of singularities. Instead, the wavelet transforms can provide localisation in both the time and frequency domains by means of compactly supported wavelets and can therefore measure the local Lipschitz exponent at a particular location. The continuous wavelet transform (CWT) is preferred since it calculates the wavelet coefficients at every possible scale and time instant and hence detects more precisely the location of singularities in the time-scale domain. In contrast, the discrete wavelet transform (DWT) uses discretisation methods to obtain the scale and time parameters.

The CWT of a time-dependent signal $f(t)$ is defined by the convolution between the signal $f(t)$ and the scaled and time-shifted wavelet $\psi(t)$, as described below:

$$
W_{\psi} f(s, \tau)=|s|^{-1 / 2} \int_{-\infty}^{+\infty} f(t) \psi\left(\frac{t-\tau}{s}\right) d t
$$

where $s$ is a scaling factor controlling the amplitude and the duration of the wavelets and $\tau$ represents a time instant shifting on the time axis.

Suppose the wavelet $\psi(t)$ and the signal $f(t)$ to be analysed are both real, then local modulus maxima at point $\left(s_{0}, \tau_{0}\right)$ can be defined such that $\left|W_{\psi} f\left(s_{0}, \tau\right)\right|<\left|W_{\psi} f\left(s_{0}, \tau_{0}\right)\right|$, where $\tau$ is either the right or left neighbourhood of $\tau_{0}$. Therefore, at a given scale $s_{0}$, a series of modulus maxima can be obtained from wavelet coefficients $W_{\psi} f\left(s_{0}, \tau\right)$. In the time-scale plane, maxima lines are defined as those curves that are connected by arbitrarily close points across the scales, along which all points are modulus maxima. The existence of maxima lines indicates the existence of local edges or sharp transitions in the signal. Such singularities can be calculated by the decay of wavelet coefficients along the maxima lines when the scale $s$ approaches zero at the certain point $\tau_{0}$.

Assume that there exists a scale $s_{0}>0$ and a constant $C$, such that for $s<s_{0}$ all the modulus maxima of $W_{\psi} f(s, \tau)$ belong to a cone defined by $\left|s-s_{0}\right|<C s$. Then, the function $f(t)$ is Lipschitz $\alpha$ at $\tau_{0}$ if, and only if, there exists a constant $A$, such that at each modulus maxima within the cone:

$$
\left|W_{\psi} f(s, \tau)\right|<A s^{\alpha+0.5}
$$

Equation (2) offers a straightforward method to calculate the Lipschitz exponent $\alpha$ of the singular points. On a logarithmic scale, the Lipschitz $\alpha$ at $\tau_{0}$ can be obtained by finding the slope of the linear relationship, as follows:

$$
\log \left|W_{\psi} f(s, \tau)\right|<\log A+(\alpha+0.5) \log s
$$

The selection of an appropriate wavelet and the scales are crucial for applications. For a practical signal, it can be written as a polynomial when expanded into a Taylor series:

$$
f(t)=\sum_{0<m<k} c_{m} t^{m}
$$

Wavelets are usually designed with vanishing moments. A wavelet $\psi(t)$ is said to have $k$ vanishing moments if it satisfies the condition:

$$
\int_{-\infty}^{+\infty} t^{j} \psi(t) d t=0 \quad j=0,1,2, \ldots, k-1
$$

If a wavelet $\psi(t)$ has $k$ vanishing moments, such a wavelet is orthogonal to polynomials of degree $k-1$. Consequently, the wavelet transform, as described in Equation (1), can remove the polynomial trends and detect singularity points in signals. Essentially, if $\psi(t)$ has one vanishing moment, the modulus maxima appears at signal discontinuities; if $\psi(t)$ has two vanishing moments, the modulus maxima also corresponds to discontinuities on the derivative of the smoothed signal. This implies that the number of modulus maxima, and hence the number of maxima lines across the entire time-scale space, increases linearly with the number of vanishing moments of the wavelet. Therefore, the wavelets to be selected are those with as few vanishing moments as possible, but with enough moments to detect the discontinuities that are of interest in the signal.

In this study, the Gaussian wavelet with one vanishing moment is used since it is sufficient to detect discontinuities appearing in the measurement signal. For example, the Lipschitz exponent $\alpha$ of a Dirac signal is estimated as 0 , whereas the $\alpha$ of a signal that is differentiable at a time instant is measured as 1 . The Gaussian function $g(t)$ can be represented by Equation (6), where $\sigma$ is the standard deviation:

$$
g(t)=\frac{1}{\sqrt{2 \pi} \sigma} e^{\left(-\frac{t^{2}}{2 \sigma^{2}}\right)}
$$

The Gaussian wavelet with one vanishing moment is defined as the first-order derivative of $g(t)$. The Fourier transform of the first order derivative of the Gaussian function is:

$$
\hat{G}(\omega)=(j \omega) e^{-\frac{\omega^{2}}{2}}
$$

As can be seen from Equations (6) and (7), the Gaussian wavelet is perfectly local in both the time and frequency domains.

Generally, modulus maxima at lower scales (high frequencies) are affected by noise components in the original signal. In contrast, the wavelet transform at higher scales (lower frequencies) have a poorer time resolution; therefore, the maxima lines may contain the modulus maxima of their adjacent points. Once the scale range is determined, the maxima lines within the selected scale region are 
then used to estimate the Lipschitz $\alpha$ with the least-squares method, as given in Equation (3).

The wavelet modulus maxima method has been used to infer the original site of the partial discharge source by investigating pulse propagation along the induction motor winding ${ }^{[9]}$, to detect transient travelling waves for directional transmission line protection ${ }^{[18]}$ and to locate faults for ageing underground cable systems $^{[19]}$

\subsection{FPGA implementation}

Many of the most popular FPGAs are in-system programmable, allowing modification of the operation of the device for dedicated applications ${ }^{[20]}$. The target FPGA in this system is a Virtex-5 LX30 FPGA from Xilinx, working at an operating clock frequency of $90 \mathrm{MHz}$. It contains $19.2 \mathrm{k}$ flip-flops, $19.2 \mathrm{k}$ look-up tables and $1152 \mathrm{kbit}$ embedded block RAM. This allows a data stream of 32k elements to be processed at one time. Figure 2 shows the flowchart for calculation of wavelet-based Lipschitz exponents. As can be seen from Section 2.2, calculation of the Lipschitz exponent involves huge matrix manipulation and has its own computational complexity due to the use of a CWT transform to obtain local modulus maxima. It is therefore necessary to implement it using a combination of algorithms on a real-time processor in the control unit and the FPGA.

The time taken for performing FFT analysis on a data stream of, for example, $10^{4}$ elements is $3.9 \mathrm{~ms}$ on the FPGA, compared to $93.7 \mathrm{~ms}$ using the real-time processor (a $533 \mathrm{MHz}$ MPC8347 processor with $256 \mathrm{MB}$ RAM) and $80.7 \mathrm{~ms}$ on the host PC (the CM server with a $2.93 \mathrm{GHz}$ Intel Core 2 Duo processor and 4 GB RAM), respectively. It can clearly be seen that the FPGA-based hardware processing unit considerably outperforms both the host computer and the real-time processor.

The condition monitoring algorithm has been implemented in real-time with the reconfigurable hardware system described above, in this case using National Instruments Compact RIO (cRIO) hardware consisting of a real-time control unit attached to an $\mathrm{I} / \mathrm{O}$ chassis. Monitoring signals are generated in real-time by a dSPACE control board, operating in lieu of sensors on a wind turbine. These signals are fed into analogue input modules housed in the I/O chassis and the Xilinx FPGA embedded within the chassis manages the signal acquisition and processing tasks. The hardware set-up is shown in Figure 3, comprising (from left to right) the host PC, the cRIO control unit and attached I/O chassis and the dSPACE control board. The cRIO hardware, including the FGPA, is programmed using LabVIEW.

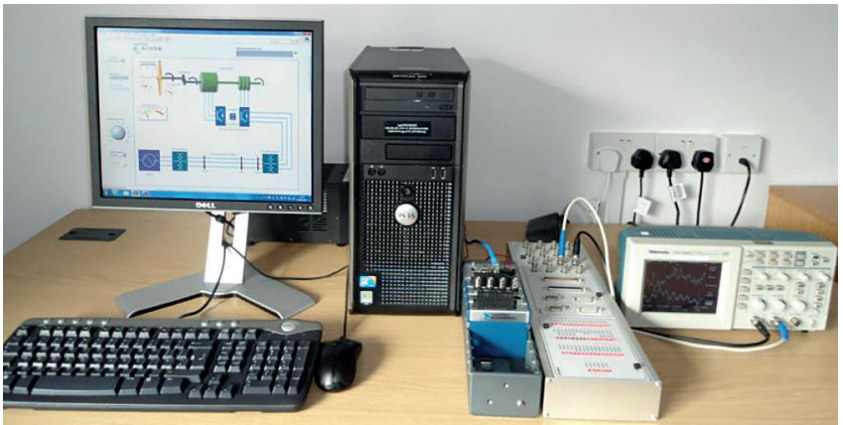

Figure 3. Reconfigurable hardware used to implement the proposed singularity detection algorithm

The dSPACE software is used to produce signals from text files created by the wind turbine simulations. These signals are generated by the dSPACE control board in real-time at a sample rate of $5 \mathrm{kHz}$. The signals are subsequently fed into the analogue input modules and the FPGA passes the signals to the cRIO control unit via a local bus. The singularity detection method is undertaken by a real-time processor housed within the control unit on a sliding window of 500 data points. The corresponding Lipschitz exponents are calculated by the FPGA and the real-time processor, as outlined previously.

\section{Validation of algorithms}

\subsection{Wind turbine simulation}

The performance of wind turbines can be monitored via the analysis of measured signals in order to obtain insight into developing faults well ahead of damage affecting the system. However, it is difficult to obtain such practical condition monitoring data from real wind turbines. Data from the simulation of distributed generation power systems, to which empirical faults can be applied, can be used to test and validate the proposed condition monitoring algorithms.

The wind turbine simulation used in this paper has been constructed using Simulink/ SimPowerSystems (SPS) and is based upon a model developed by researchers at Hydro-Québec. This simulation comprises a 1.5 MW wind turbine connected to a $30 \mathrm{~km}$ $25 \mathrm{kV}$ transmission line, exporting power to a $120 \mathrm{kV}$ grid, as illustrated in Figure 4 . The transmission line is divided into several sections in order to incorporate faults at a range of distances from the point of common coupling (PCC) of the wind turbine to the power network, with switches to allow the fault location to be selected. Further switches allow the selection of the fault type, such as AG (phase-A-to-ground), AB (phase-A-to-

Figure 2. The flowchart for Lipschitz exponent calculation 


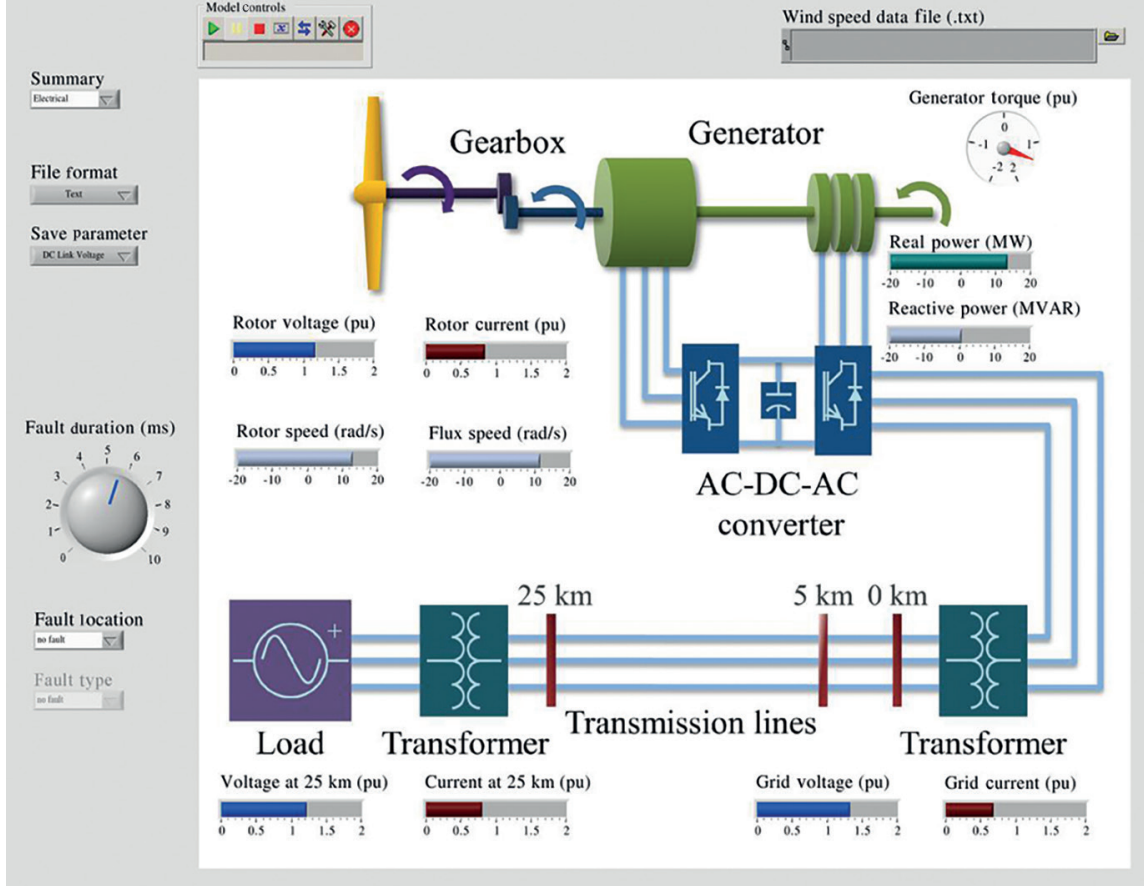

Figure 4. User interface of the SimPowerSystems model for DFIG wind turbine simulations

phase-B short circuit), ABG (phase-A-to-phase-B-to-ground) or ABCG (three-phase-to-ground). The $120 \mathrm{kV}$ network load model is represented by an ideal voltage source and equivalent system impedance. The transformers used to connect the wind turbine to the transmission line and the transmission line to the grid are $575 \mathrm{~V}$ to $25 \mathrm{kV}$ and $25 \mathrm{kV}$ to $120 \mathrm{kV}$, respectively. The electrical subsystem of the wind turbine model is of a DFIG type. The AC-DC-AC converter comprises rotor- and grid-side converters and a capacitor is connected on the DC side to ensure variations in the DC-link voltage are kept to a minimum. The converters are regulated by pulse width modulation (PWM) controllers. A coupling inductor is used (not shown in Figure 4) to filter the high-frequency component of the voltage before the grid-side converter is connected to the grid.

The control system for the turbine comprises three coordinated controllers. The rotor-side controller regulates the power output of the wind turbine and the voltage (or reactive power) measured at the machine stator terminals. This is achieved by adjusting the torque or by regulating the speed of the generator. The power is controlled to follow a predefined turbine power-speed characteristic. The grid-side controller principally maintains the DC link voltage regardless of the direction and magnitude of the rotor power. Moreover, it allows the exchange (generation or absorption) of reactive power to the network for voltage support requirements, as required. Finally, the pitch angle controller regulates the speed of the generator at high wind speeds: when the nominal generator power is reached, the pitch angle controller limits the rotor speed by adjusting both the pitch angle and its rate of change.

In this system, a host PC with a $2.93 \mathrm{GHz}$ Intel Core 2 Duo processor was used for modelling and simulation of the wind turbine and transmission system. The simulation is controlled using a graphical user interface developed in LabVIEW on the host computer to allow interaction with the simulation. Following compilation of the Simulink model as a real-time library file, it is incorporated in LabVIEW. Controllers and indicators are assigned to the variables defined in the Simulink model.

\subsection{Detection of grid faults}

The detection of grid faults is used to demonstrate how far a grid fault can still be detected at the point of common coupling of the wind turbine, thereby examining the influence of the grid fault on the turbine's operational performance. In order to demonstrate how grid faults can be detected with the early warning condition monitoring algorithms, typical asymmetrical and symmetrical faults, such as AG and ABCG faults, have been introduced individually into the simulation after $5 \mathrm{~s}$, each lasting $0.2 \mathrm{~s}$. Root-mean-square (RMS) values of the signal have been proven to be more suitable for condition monitoring as they contain more critical features than instantaneous values ${ }^{[21]}$. Figure 5 illustrates the RMS phase current output for an AG fault and an ABCG fault occurring at different locations between $0 \mathrm{~km}$ and $30 \mathrm{~km}$ from the point of connection. Note that the ABCG grid fault exhibits a distinctive double peak trace. The faults become less detectable due to the reduction in magnitude of the measurement signal when the fault is

\section{Grid AG faults}

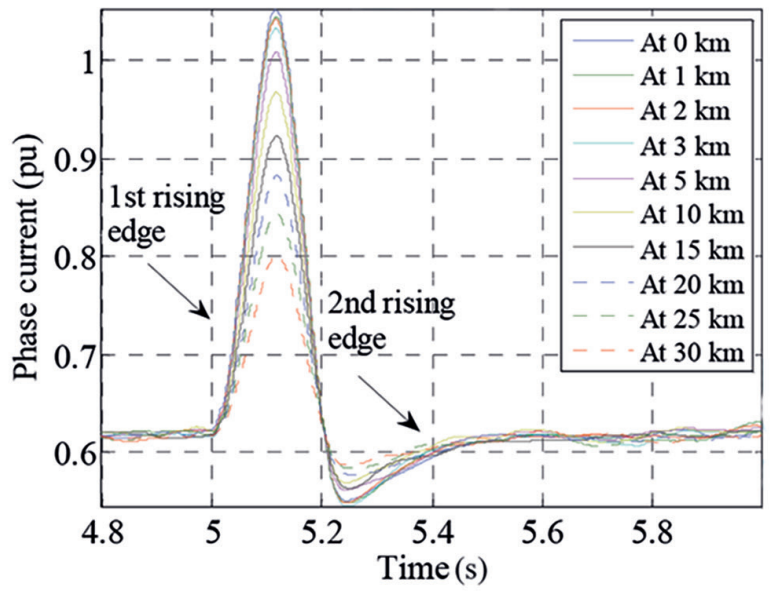

Grid ABCG faults

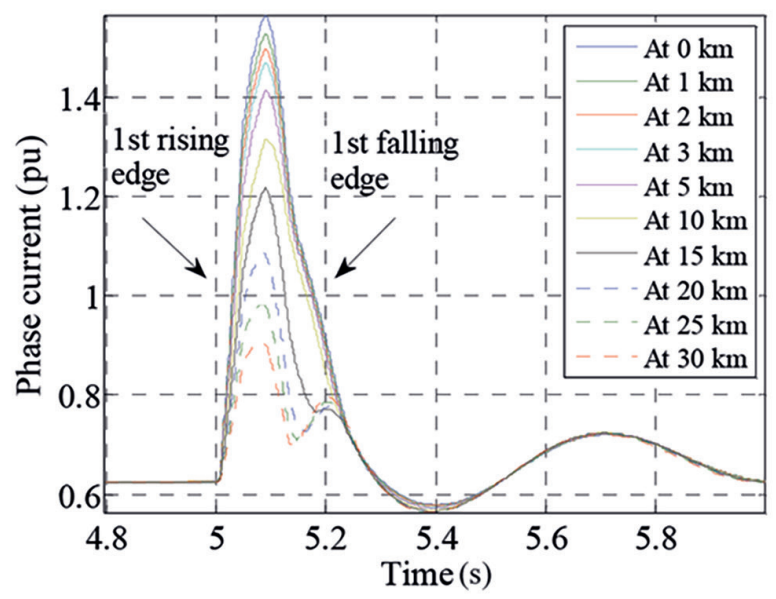

Figure 5. RMS phase current output when a fault occurs at different locations from 0-30 km: (top) AG fault and (bottom) ABCG fault 
further away from the wind turbine.

Figure 6 shows the Lipschitz exponent of the first rising edge of the phase current for both faults occurring at different locations, where, for comparison, the Lipschitz exponent of the fault-free turbine is also shown. For a healthy wind turbine, the Lipschitz exponent of the electrical signals is 1.0 because their RMS values are kept constant in the healthy conditions. Note that the maximum value corresponding to the first peak of the measurement signal versus the distance of the fault differs significantly, as can be seen from Figure 5. Nevertheless, the Lipschitz exponent value of the first rising edge of the phase current is constant, irrespective of the fault location, indicating that faults at the far end of the feeders are detectable. Lipschitz exponents resulting from the AG are larger than those produced from the ABCG. This implies that a more severe three-phase grounded fault produces much sharper transitions than those produced by a single-phase grounded fault, the most common fault type in electrical distribution networks.

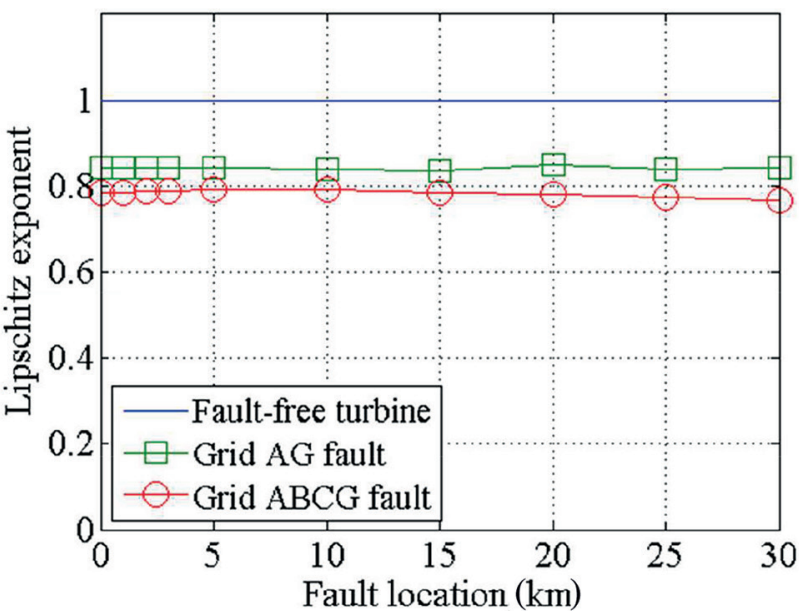

Figure 6. The Lipschitz exponent of the first rising edge of phase current due to the AG fault and the ABCG fault occurring at different locations. The Lipschitz exponent of the fault-free turbine is also shown

It is also found that, for the AG fault, the Lipschitz exponent value of the second rising edge of the phase current changes with fault location, whereas for the ABCG fault, the Lipschitz exponent value of the first falling edge of the phase current is fault-location dependent. Hence, these characteristics can be used to infer the location of the faults, even for the case where the measurement signal is weak. Here, it should be emphasised that the work presented in this paper is focused primarily on the study of early fault detection; the study of fault location is beyond the scope of this paper.

\subsection{Detection of power electronics faults}

In this section, power electronics faults are introduced into the simulation, specifically broken rotor bars and DC link capacitor faults. Rotor bar faults are associated with the thermal and magnetic stresses caused by electromagnetic forces due to the high currents carried by the bars, residual stresses from manufacturing and environmental stresses. This type of fault begins as high resistance in the rotor bars and can develop into cracks or holes ${ }^{[22]}$. As soon as cracks develop, the resistance of the bars increases further, consequently increasing localised heating and worsening the cracks. DC link capacitors are required to endure high ripple currents leading to self-heating, which, in addition to high ambient operating temperatures, can result in the deterioration of the electrolyte material and the loss of electrolyte by vapour diffusion. Furthermore, when the capacitor is operating at higher temperatures than the rated temperature, the DC voltage will be derated. The working life of a capacitor is also dependent upon operating voltage, current and frequency. Consequently, DC link capacitors, although well designed, are considered one of the weakest components employed in power converters ${ }^{[23]}$.

Rotor bar faults have been introduced into the simulation by adjusting the generator rotor phase resistances ${ }^{[24]}$. An example of simulations incorporating one to 25 broken bars is shown in Figure 7 (top), illustrating the phase current. As with the grid fault simulation, the faults occur after $5 \mathrm{~s}$ and last $0.2 \mathrm{~s}$. Figure 7 (bottom) shows the Lipschitz exponent of the first rising edge of the phase current, compared to the normalised magnitude of the first peak of the phase current for broken rotor bar faults. Indeed, the magnitude of the phase current can provide an indication of the severity of the fault. However, it can be observed that the Lipschitz exponent value of phase current is not affected by the number of broken bars, which means that a fault from one broken bar can produce an equivalent Lipschitz exponent to that produced by faults from 25 broken bars. Therefore, the rotor bar fault can be detected at an earlier stage by means of measurement of the Lipschitz exponent, before developing into an actual failure and outage.

An example of simulations incorporating a faulty DC link capacitor operating at 5\% to $90 \%$ of full capacity is shown in Figure
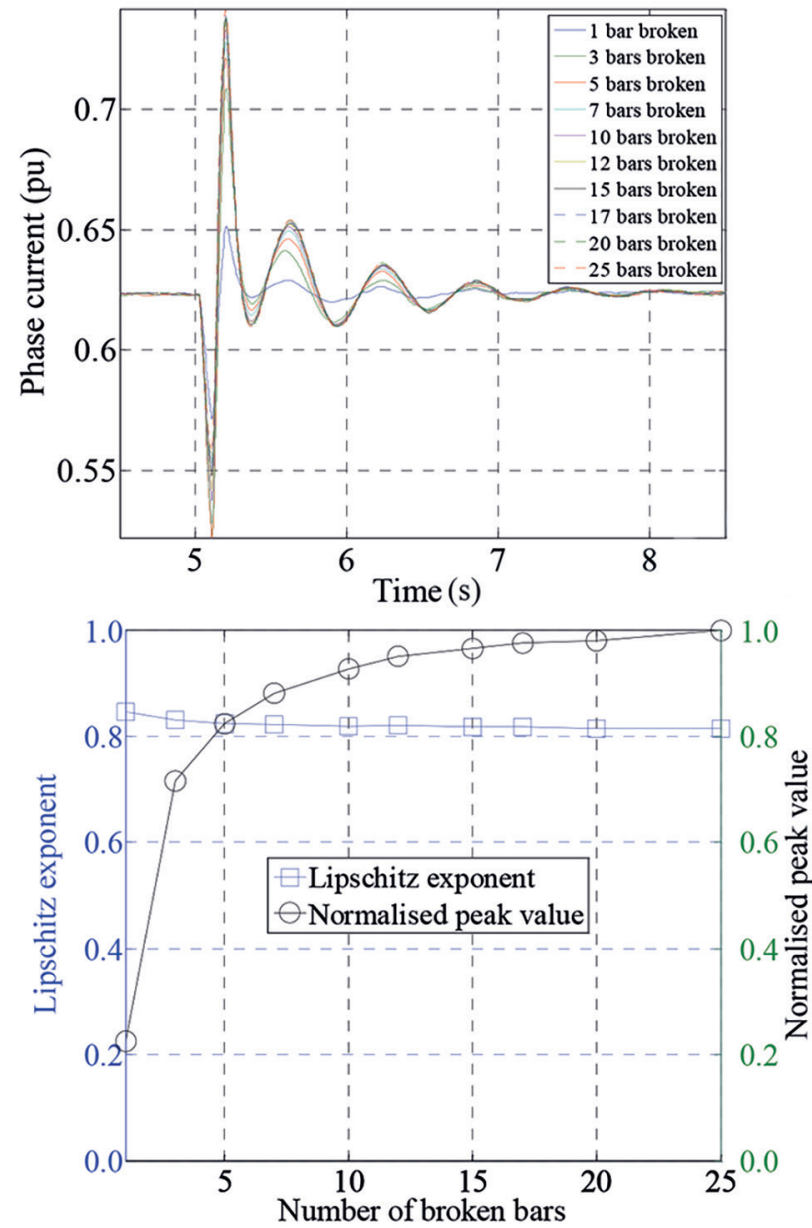

Figure 7. Detection of rotor bar faults: (top) RMS phase current output for the different number of broken rotor bars; (bottom) Lipschitz exponent value and the normalised peak value corresponding to the broken rotor bar faults 
8 (top). Similarly, the fault occurs after $5 \mathrm{~s}$ and lasts $0.2 \mathrm{~s}$. Figure 8 (bottom) shows the Lipschitz exponent of the first rising edge of the phase current in comparison with the normalised magnitude of the first peak of the phase current for DC link capacitor faults. Again, in contrast to the trend visible for the magnitude of the peak, the Lipschitz exponent value is little affected by the capacitance drops. As a result, the capacitance loss in the DC link capacitor can be detected at an earlier stage when using the Lipschitz exponent method.
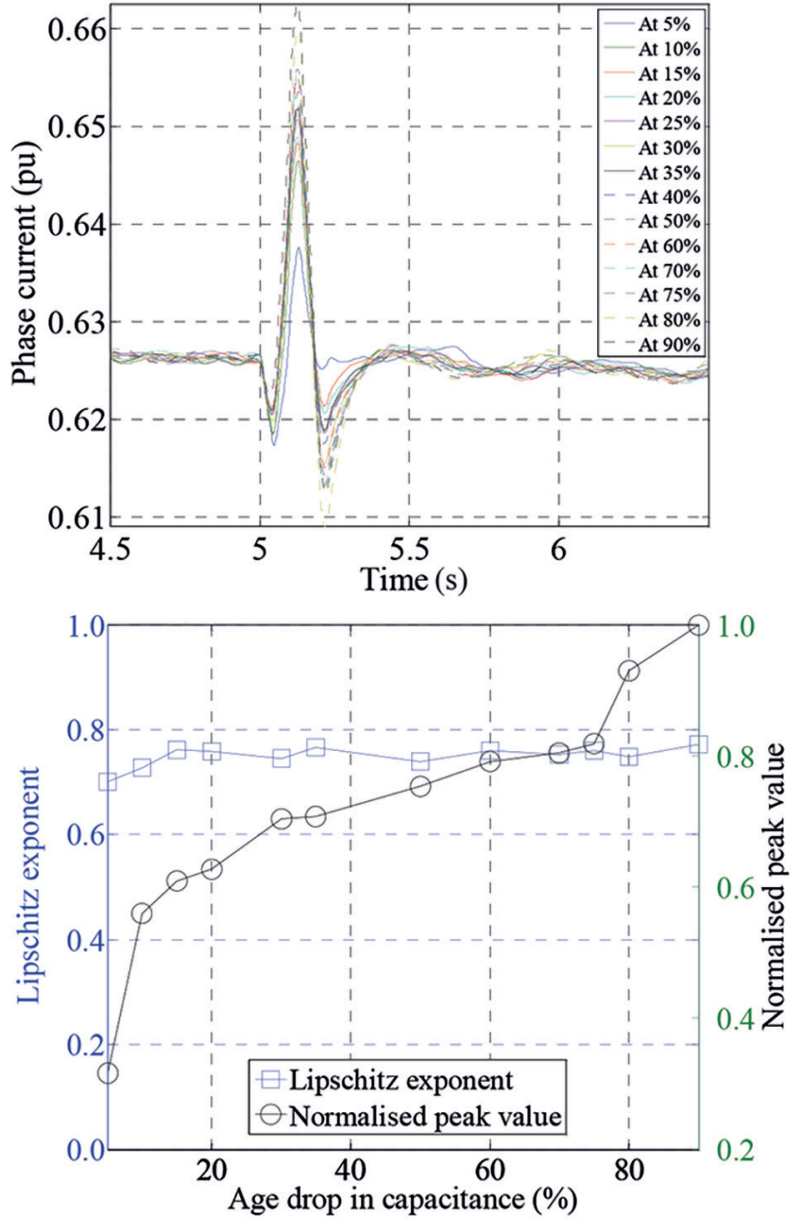

Figure 8. Detection of DC link capacitor faults: (top) RMS phase current output for the different age drop in capacitance of DC link capacitor; (bottom) Lipschitz exponent value and the normalised peak value corresponding to the capacitance drops in DC link capacitor

\section{Conclusions}

This paper has presented a novel electrical condition monitoring system for wind turbines. The reconfigurable structure can provide flexibility of the CM system to monitor a specific set of turbine components by adding new hardware modules and data analysis software. In order to provide an early warning indicator, a method for extracting local geometrical features represented by the local singularities of a signal is proposed. Using this wavelet-based singularity detection method, a fault signal of small magnitude generated at the early stage of a fault was proven to carry the same Lipschitz exponent value as the signal of large magnitude at the late stage of the fault. The relationship between the Lipschitz exponent of the measurement signal and the location and severity of faults occurring on the grid and in the power electronics was discussed. The work demonstrates that the technology can be used to provide an early warning before a fault develops into a detrimental one.

Electrical condition monitoring systems normally gather highfrequency and instantaneous data for analysis. Consequentially, when implemented online, it is essential for the system to process these data in real time. The FPGA employed in the proposed system allows the real-time implementation of digital signal processing tasks, ie the wavelet modulus maxima algorithms in this study, and offers the ability to run multiple tasks concurrently.

The proposed method is essentially immune to noise. The distribution of the modulus maxima of noise generally concentrates on low scales, ie higher frequencies, which decay quickly and cannot propagate further. If there are still maxima lines generated by noise within the selected scale range, their Lipschitz exponents are negative in contrast to positive ones corresponding to the signal discontinuities. In the present study, only one of the distinctive rising or falling edges was used for Lipschitz exponent measurement; however, there are in fact a number of rises and falls in the signal that can be useful for analysis, depending on the type of fault. Further work will incorporate Lipschitz values with statistical moments of the coefficient modulus maxima in specific scales or frequency bands to further identify the location, duration and severity of the faults.

The technique has been demonstrated using data obtained from computer simulations of a wind turbine with a grid connection. It is worth noting that it is difficult to obtain practical condition monitoring data containing different types of fault in wind turbines, particularly when the faults are in an early stage of development. Future work will investigate the Lipschitz exponent for a signal from a fault-free wind turbine operating in variable wind conditions as the detection baseline and compare the value of the exponent for different fault types. Future work will also seek to use condition monitoring data obtained from the controllable experimental tests and from real wind turbines to demonstrate the proposed early warning fault detection technique.

\section{Acknowledgements}

The authors would like to thank the UK Engineering and Physical Sciences Research Council (EPSRC) for their funding support of the project under Grant EP/I037326/1.

\section{References}

1. C S Gray and S J Watson, 'Physics of failure approach to wind turbine condition-based maintenance', Wind Energy, Vol 13, pp 395-405, 2009.

2. C A Walford, 'Wind turbine reliability: understanding and minimising wind turbine operation and maintenance costs', Technical Report, SAND 2006-1100, Sandia National Laboratories, 2006.

3. G J W Van Bussel and C Schöntag, 'Operation and maintenance aspects of large offshore wind farms', Proceedings of the European Wind Energy Conference (EWEC'97), Dublin, Ireland, 1997.

4. P J Tavner, 'Review of condition monitoring of rotating electrical machines', IET Electric Power Applications, Vol 2, No 4, pp 215-247, 2008.

5. Z Hameed, S H Ahn and Y M Cho, 'Practical aspects of a condition monitoring system for a wind turbine with emphasis on its design, system architecture, testing and installation', Renewable Energy, Vol 33, pp 879-894, 2010. 
6. Z Hameed, Y S Hong, Y M Choa et al, 'Condition monitoring and fault detection of wind turbines and related algorithms: a review', Renewable and Sustainable Energy Reviews, Vol 13, pp 1-39, 2009.

7. M L Wymore, J E Van Dam, H Ceylan and D Qiao, 'A survey of health monitoring systems for wind turbines', Renewable and Sustainable Energy Reviews, Vol 52, pp 976-990, 2015.

8. D Raheja, J Llinas, R Nagi et al, 'Data fusion/data mining-based architecture for condition-based maintenance', International Journal of Production Research, Vol 44, pp 2869-2887, 2006.

9. X Ma, C Zhou and I J Kemp, 'Interpretation of wavelet analysis and its application in partial discharge detection', IEEE Transactions on Dielectrics and Electrical Insulation, Vol 9, pp 446-457, 2002.

10. X Ma, 'Investigations of the state-of-the-art methods for electromagnetic NDT and electrical condition monitoring, Insight: Non-Destructive Testing and Condition Monitoring, Vol 54, No 9, pp 482-488, 2012.

11. Y R Chang, C M Chan and C J Chang, 'The design of a control system and power conditioning system for a $25 \mathrm{~kW}$ active control-based wind turbine system, Proceedings of the International Conference on Power Electronics and Drive Systems, Taipei, Taiwan, pp 1272-1276, 2009.

12. J J Justo, F Mwasilu and J W Jung, 'Doubly-fed induction generator-based wind turbines: a comprehensive review of fault ride-through strategies', Renewable and Sustainable Energy Reviews, Vol 45, pp 447-467, 2015.

13. Energy Technologies Institute, 'Condition Monitoring - a report on a holistic approach to wind turbine monitoring', http://www.eti.co.uk/a-report-on-a-holistic-approach-towind-turbine-monitoring/. Accessed 8 October 2016.

14. J Rangel-Magdaleno, R Romero-Troncoso, R Alfredo OsornioRios et al, 'FPGA-based vibration analyser for continuous CNC machinery monitoring with fused FFT-DWT signal processing, IEEE Transactions on Instrumentation and Measurement, Vol 59, pp 3184-3194, 2010.

15. E Cabal-Yepez, M Valtierra-Rodriguez, R J Romero-Troncoso et $a l$, 'FPGA-based entropy neural processor for online detection of multiple combined faults on induction motors', Mechanical Systems and Signal Processing, Vol 30, pp 123-130, 2012.

16. P Cross and X Ma, 'Non-linear system identification for modelbased condition monitoring of wind turbines', Renewable Energy, Vol 71, pp 166-175, 2014.

17. J Chen, J Pan, Z Li, Y Zi and X Chen, 'Generator bearing fault diagnosis for wind turbine via empirical wavelet transform using measured vibration signals', Renewable Energy, Vol 89, pp 80-92, 2016.

18. W Chen, O P Malik, X Yin et al, 'Study of wavelet-based ultrahigh-speed directional transmission line protection', IEEE Transactions on Power Delivery, Vol 18, No 4, pp 1134-1139, 2003.

19. M Gilany, D K Ibrahim and E S T Eldin, 'Travelling wave-based fault location scheme for multi-end aged underground cable system, IEEE Transactions on Power Delivery, Vol 22, No 1, pp 82-89, 2007

20. E Cabal-Yepez, M Valtierra-Rodriguez, R J Romero-Troncoso et $a l$, 'FPGA-based entropy neural processor for online detection of multiple combined faults on induction motors', Mechanical Systems and Signal Processing, Vol 30, pp 123-130, 2012.

21. A M Gaouda and M M A Salama, 'Monitoring non-stationary signals', IEEE Transactions on Power Delivery, Vol 24, No 3, pp 1367-1376, 2009.
22. S Nandi, H A Toliyat and X Li, 'Condition monitoring and fault diagnosis of electrical motors - a review', IEEE Transactions on Energy Conversion, Vol 20, pp 719-729, 2005.

23. K W Lee, M Kim, J Yoon et al, 'Condition monitoring of DC-link electrolytic capacitors in adjustable-speed drives', IEEE Transactions on Industry Applications, Vol 44, pp 1606 1613,2008

24. S Chen and R Živanović, 'Modelling and simulation of stator and rotor fault conditions in induction machines for testing fault diagnostic techniques', European Transactions on Electrical Power, Vol 20, pp 611-629, 2010. 